\title{
En defensa de una aproximación a los conceptos emocionales desde la perspectiva de los prototipos
}

\author{
James A. Russell \\ Universidad de British Columbia
}

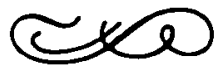

Emoción, amor, enojo, felicidad, ansiedad..., -conceptos como estos influencian la vida de la gente. Interpretamos las acciones de los otros y los estados temporales a través de estos conceptos, y orientamos nuestra conducta de acuerdo a ellos. Un acto realizado en medio de un estado emocional tiene un status legal diferente que el mismo acto. llevado a cabo de manera calmada. Nos preguntamos, «¿es esto realmente amor?» o «¿aún lo estoy queriendo?». Según Schachter y Singer (1962): y Harré (1987), tener una emoción depende de cómo nos etiquetemos en los términos de enojo o felicidad, etc. (Los términos en cursiva indican conceptos o palabras que expresan dichos conceptos.) Los psicólogos utilizan esas mismas palabras al comunicarse con sus pacientes o sujetos, y al encuadrar las hipótesis para la investigación. Los psicológos necesitan comprender los conceptos emocionales en nuestro lenguaje natural; estos conceptos (considerados distintos de los acontecimientos emocionales) son el tema de este artículo.

Agradecimientos: Este artículo está basado en investigaciones financiadas por la Social Sciences and Humanities Research Council of Canada. Este artículo contiene material procedente de artículos previos.

Aradezco a Martin A. Conway, Beverly Fehr, P. N. Johnson-Laird, William Lyons, Michael F. Mascolo y Keith Oatley sus comentarios sobre las cuestiones consideradas en este artículo.

Publicado originalmente: En inglés en el Joumal of Personality and Social Psychology, 1991, 60, 37-47. Copyright (C) 1991 por la American Psychological Association. Traducido con permiso del editor y el autor. La American Psychological Association no se responsabiliza de la calidad de esta traducción. Ni el original ni esta traducción pueden publicarse de nuevo, fotocopiarse o reimprimirse sin una autorización expresa y por escrito de la American Psychological Association.

Traducción: Darío Páez Rovirá.

Dirección del autor: James A. Russell, Department of Psychology, University of British Columbia, Vancouver, BC, Canada V6T $1 Y 7$. 
Una comprensión de estos conceptos, creo, se puede llevar mejor a cabo en la actualidad a través de la perspectiva de los prototipos que a través de la aproximación clásica, y un buen número de investigadores basan su trabajo en este punto de partida. Chaplin, John y Goldberg (1988), por ejemplo, argumentan que la diferencia real entre un estado, incluyendo una emoción, y otros conceptos psicológicos como rasgos de personalidad, se pueden entender perfectamente desde una perspectiva prototípica. La teoría de los prototipos nos ha dado «insights» sobre otros conceptos centrales de la psicología, incluyendo actos conductuales (Buss y Craik, 1983), rasgos de personalidad (Cantor y Mischel, 1979), inteligencia (Neisser, 1979), situaciones sociales (Cantor, Mischel y Schwartz, 1982) y establecimiento ambiental (Tversky y Hemenway, 1983). Además, la teoría ha inspirado importantes nuevas aproximaciones en el diagnóstico psiquiátrico (Cantor, Smith, French y Mezzich, 1980) y en la medición de la personalidad (Broughton, 1984).

Por otro lado, la perspectiva de los prototipos ha sido objeto de debate (Armstrong, Gleitman y Gleitman, 1983; Hanard, 1987; Lakoff, 1987; Lysak, Rule y Dobbs, 1989; Osherson y Smith, 1981) y se le han planteado críticas específicas en el estudio de los conceptos emocionales (Clore y Ortony, 1988; JohnsonLaird y Oatley, 1986, 1988, 1989; Ortony, Clore y Foss, 1987). En este artículo me voy a limitar a analizar los conceptos emocionales. Mi objetivo es delinear un análisis de dichos conceptos, a través de la línea de los prototipos, revisar en su conjunto las críticas a la perspectiva de los prototipos y defender el análisis presentado aquí contra estas críticas. Ortony et al. (1987) y Johnson-Laird y Oatley $(1986,1988,1989)$ presentaron sus críticas a la perspectiva de los prototipos en el contexto de la exposición de sus propios análisis. Esto creó la impresión de un conflicto entre estos puntos de vista. En una sección final, voy a sugerir que las dos posiciones en este debate pueden haber estado hablando de manera cruzada y, por tanto, pueden ser reconciliadas. El objetivo es, de ahora en adelante, los comentarios y las críticas a la perspectiva de los prototipos, más que sus propias propuestas.

\section{EL PUNTO DE VISTA CLASICO}

Los términos de definición y definición clásica se utilizan a menudo como si cualquiera comprendiera claramente lo que significan. Pero cada autor puede significar algo diferente o se pueden plantear las definiciones clásicas para diferentes usos. Voy a empezar considerando el punto de vista clásico, según como se aplica a los conceptos de lenguaje natural, más que a los conceptos técnicos. Los conceptos del lenguaje natural son los que subyacen al uso cotidiano de términos comunes como emoción, enojo y amor. Desde el punto de vista clásico, conocer la significación (la intensión) de cada uno de estos términos, captar el concepto asociado a ellos, es conocer, al menos implícitamente, un conjunto de rasgos necesarios y suficientes. Cada término común se refiere a un conjunto de objetos o acontecimientos (la extensión) cuyos miembros - cada uno de ellosposee estos atributos. El objeto o acontecimiento es un miembro de la categoría nombrada por el término, en virtud de poseer esos atributos.

El punto de vista clásico se interesa por lo que es necesariamente verdadero, más que por lo que pasa por ser verdadero. Por ejemplo, las abuelas a menudo tienen el pelo gris, pero no necesariamente. Las abuelas son, también, madres 
de un padre o madre; esto es necesario y se comprende por el simple conocimiento del significado de los términos implicados. Así, el conocimiento de los atributos definitorios permite a un sujeto realizar ciertas operaciones lógicas con un término.

Para presentar el punto de vista clásico en otras palabras, tenemos la caracterización de Markman (1989, p. 5).

«Las definiciones clásicas requieren que las categorías tengan una intensión y una extensión que determinen la una a la otra. La intensión de una categoría es el conjunto de atributos o rasgos que definen la categoría; a veces ello es considerado como el significado del término de la categoría... La extensión de una categoría es el conjunto de objetos que son miembros de la categoría, esto es, el conjunto de objetos que reúnen el conjunto de criterios descritos en la definición intensional».

En los comentarios que siguen, es importante mantener presente esta distinción entre palabras y conceptos, por un lado, y los hechos a los cuales se refieren las palabras, por el otro. Para los propósitos de la primera sección de este artículo voy a asumir que los que atacan el punto de vista de los prototipos y defienden el punto de vista clásico consideran el punto de vista clásico tal y como lo he planteado previamente. Más adelante voy a considerar otra posibilidad en lo concerniente al punto de vista clásico, no a las palabras cotidianas y sus conceptos asociados, pero sí a los conceptos técnicos definidos por los expertos en sus esfuerzos por comprender los acontecimientos referidos por las palabras cotidianas.

\section{Síntesis de un análisis prototípico de los conceptos de emoción}

A pesar de los siglos de esfuerzos, de los filósofos primero y más tarde de los psicólogos, la aproximación clásica todavía no logra obtener una definición compartida para los conceptos como emoción, enojo, amor y otros parecidos. Tampoco se detectó ningún signo de progreso. William James (1902/1929, 1890/1950) se declaró escéptico respecto a la posibilidad de una definición clásica, este escepticismo encontró eco en Duffy $(1934,1941)$, Kagan (1978) y Averill $(1980,1982)$. Autores de diversas disciplinas también se han mostrado escépticos, en general, sobre la visión clásica (Labov, 1973; Lakoff, 1987; Kay y McDaniel, 1978; Zadeh, 1965). Los análisis filosóficos de Wittgenstein, sobre los juegos, son el argumento más famoso en contra de la visión clásica, pero gran parte de la investigación psicológica actual complementa sus argumentos (Mervis y Rosch, 1981; Smith y Medin, 1981).

Defender la aproximación prototípica frente a la aproximación clásica a los conceptos no significa plantear que los conceptos no puedan ser definidos; los conceptos son sistemáticos y ordenados, pero están organizados alrededor de diferentes planteamientos que los asumidos por la versión clásica. Rosch (1987) planteó una alternativa a la visión clásica, lo que condujo a una competencia saludable entre puntos de vista no clásicos (Kahneman y Miller, 1986; Lakoff 1987; Neisser, 1987; Smith y Medin, 1981). Para un punto de vista, los conceptos no son inherentemente vagos, pero varían de una persona a la otras, y para la misma persona entre un momento o un contexto y otro (Barsalou, 1987). Lo que me preocupa en este artículo no tiene que ver con las ventajas o desventajas de las diferentes alternativas en el seno de la familia de los modelos no-clásicos. (El nombre que Medin, 1989, le da a esta familia es el de «probabilística», quizás más adecuado que el de «prototípica» que estoy usando en el título de este 
artículo.) Sin embargo, me voy a centrar en los atributos que son comunes a la mayoría de las aproximaciones no clásicas y que las distinguen de una aproximación clásica a los conceptos.

Mis colaboradores y yo hemos ofrecido un análisis de las palabras inglesas para la emoción, desde la perspectiva de los prototipos (Bullock y Russell, 1986; Fehr, 1982; Fehr y Russell, 1984; Fehr, Russell y Ward, 1982; Russell, 1989; Russell y Bullock, 1986). Otros investigadores también han encontrado útiles las ideas de los prototipos en sus análisis de los conceptos emocionales (Averill, 1980; Burch y Pischkin, 1984; Conway y Bekerian, 1987; Gurtman, 1987; Horowitz, Wright, Lowenstein y Parad, 1981; Iaccino, 1989; Lakoff, 1897; Mascolo y Mancuso, 1988; Shaver, Schwartz, Kirson y O'Connor, 1987; Shields, 1984; Tiller y Harris, 1984). Se han empezado a realizar análisis prototípicos de otros léxicos emocionales, además del inglés (Bormann-Kischkel, Hildebrand-Pascher y Stegbauer, 1990; Rombouts, 1988), pero lo importante es que la aproximación prototípica no presupone que los conceptos del lenguaje inglés sean universales (Lutz, 1985; Russell, 1989).

Tanto los análisis clásicos como prototípicos de los conceptos emocionales dibujan este dominio como una jerarquía inclusiva, como se muestra en la Figura 1. En un nivel superior está la palabra emoción. En el nivel intermedio, la palabra emoción se divide en miedo, enojo, felicidad y otras. Muchas de las categorías de este nivel pueden ser, a su vez, subdivididas, formando un nivel subordinado. Por ejemplo, a enojo podemos dividirlo en rabia, disgusto, etc.

De acuerdo a la visión clásica, existirá un conjunto de categorías en cada nivel jerárquico; en algunas versiones, las categorías de un nivel son mutuamente excluyentes, aunque el miedo y el enojo, por ejemplo, pueden coexistir, ninguna emoción es simultáneamente miedo y enojo.

\section{FIGURA I}

Una jerarquía inclusiva de emoción

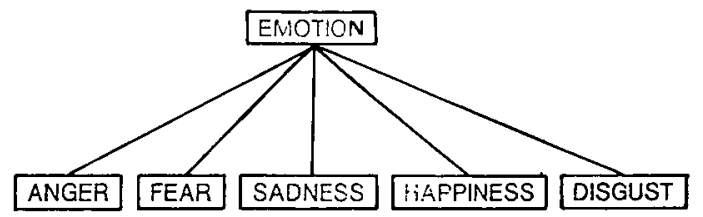

Por el contrario, según el punto de vista de los prototipos, esta jerarquización no es más que una aproximación a las relaciones de inclusión que existen dentro del ámbito emocional. Por ejemplo, en el nivel prototípico medio de las emociones aparecen emociones menos prototípicas que se dibujan en no emociones, sin encontrar fronteras claras entre unas y otras. Este nivel contiene entonces un número indeterminado de categorías, desde el enojo hasta el disgusto ligero. Por la misma razón, el número de subcategorías dentro de las categorías del nivel medio es indeterminado. A un nivel dado, las categorías no son mutuamente excluyentes, sino que se sobreponen las unas a las otras.

En el centro de la perspectiva de los prototipos está la idea de que ser miembro de una categoría está determinado por el parecido. La idea de parecido es utilizada aquí en un sentido general lo suficientemente amplio como para que incluya el punto de vista de la similitud, basado en el conocimiento, que suscribe Medin (1989). 
Los objetos concretos o los acontecimientos son considerados miembros de una categoría por su parecido suficiente a lo que podemos llamar ejemplares prototípicos. Esto es, la representación mental de un objeto o acontecimiento factua se compara con la representación mental de los ejemplares prototípicos de la categoría. El parecido es un asunto de grados, los miembros de la categoría varían en la extensión a la que pertenecen (se dice que la categoría tiene una estructura interna); no hay fronteras claras que separen a los miembros de los no miembros (las categorías tienen fronteras sutiles); y los miembros se parecen los unos a los otros, superponiéndose y entrecruzándose en formas que varían en tipo y número (los miembros de la categoría comparten un parecido de familia). Compárense estas propiedades con la visión clásica en la que ser miembro de una categoría está determinado por una serie de rasgos o atributos comunes: todos los no miembros son iguales en su pertenencia a la categoría $\mathrm{y}$ todos los miembros pueden ser distinguidos en forma precisa de los miembros.

Existen diferentes hipótesis desde la perspectiva no clásica en los referente a la naturaleza de los ejemplares con los cuales los acontecimientos y objetos son comparados. En algunas exposiciones, los hechos y los objetos se comparan en relación al recuerdo de experiencias individuales concretas (Kahneman y Miller, 1986); en otras argumentaciones se comparan a esquemas generalizados: ideales hipotéticos sencillos (Neisser, 1979); ejemplares promedios (Posner y Keele; 1968); o puntos de referencia (Rosch, 1977). Las palabras caso paradigmático, estereotipo y prototipo también han sido utilizadas (véase Barsalou, 1985, para una comparación de algunas de estas hipótesis).

Para los conceptos emocionales, una posibilidad planteada es la de un script (Abelson, 1981). Un guión o script es a un acontecimiento lo que un prototipo es a un objeto. El acontecimiento es pensado como una secuencia de subacontecimientos. Aunque a menudo hablamos de la emoción como de una cosa, una descripción más útil es plantearla como una secuencia de subacontecimientos. En otras palabras, los atributos que constituyen los conceptos emocionales describen los subacontecimientos que constituyen una emoción: causas, creencias, sentimientos, cambios fisiológicos, deseos, acciones abiertas, expresiones vocales y faciales. Estos subacontecimientos, descritos por los atributos del concepto, son ordenados en una secuencia casual -en una forma muy similar a como las aciones se ordenan en el guión de una obra de teatro-. Conocer el sentido de un término como enojo, miedo o celos, es conocer el script o guión de esta emoción. La hipótesis presentada aquí es el significado de cada uno de esos términos, el concepto que expresa cada término es un script (1). Sin embargo, pocos o ninguno de los atributos del guión son necesarios; más aún, cuantos más atributos están presentes más grande es el parecido y más apropiado el etiquetado del script.

A modo de ejemplo, la Tabla 1 describe un posible script para el enojo. La secuencia descrita en este cuadro puede no haber ocurrido nunca, pero conocemos una secuencia como ésta para cada concepto emocional. El script nos permite interpretar nuestras acciones y las de los demás. Un acontecimiento real se examina como una actualización posible del script. El acontecimiento factual puede parecerse al script de diferentes maneras y grados. Además, cada atributo del script puede tener un valor óptimo, a veces típico, a veces ideal, que sirve como un valor por defecto. Los atributos y los subacontecimientos de los acontecimientos en el mundo pueden parecerse a los valores de los atributos del script, también en diferentes formas y grados. 
TABLA 1

El script de enojo

1. La persona es ofendida. La ofensa es intencional y dañina. La persona es inocente. Se ha cometido una injusticia.

2. La persona mira fijamente al ofensor y frunce el ceño.

3. La persona siente una tensión interna y agitación, como si la presión y el calor estuvieran incrementándose rápidamente dentro de su cuerpo. Nota latir su corazón y la tensión de sus músculos.

4. La persona desea que se haga justicia.

5. La persona pierde el control y golpea, dañándolo, al ofensor.

NOTA:

Este script de enojo está parcialmente basado en el análisis del enojo de Lakoff (1987).

La noción de script nos ayuda a explicar por qué ciertos casos son noemocionales o fronterizamente emocionales, a pesar de la presencia de los atributos que a menudo se plantean como definitorios. Por ejemplo, si enojo fuera definido por la activación fisiológica, por una mueca facial y por una tendencia a agredir; si miedo fuera definido por la activación fisiológica, por un ceño fruncido y una tendencia a huir, entonces, por definición, un boxeador podría ser un claro caso de enojo y un corredor un claro caso de miedo. Eștos atletas son malos ejemplos de estas emociones porque carecen del contexto y los antecedentes típicos.

En una serie de estudios, se ha explorado empíricamente la aplicabilidad de la aproximación de los prototipos a los conceptos emocionales (Fehr y Russell, 1984; Fehr, Russell y Ward, 1982; Russell y Bullock, 1986). Como se hipotetizaba, se encontró que la categoría supraordenada de emoción tenía lo que Rosch llama una estructura interna: felicidad, amor, enojo, miedo, respeto, envidia y otras categorías intermedias, podían ser ordenadas de manera estable como mejores o peores ejemplos de emoción. A su vez, las puntuaciones de prototipicidad para cada término emocional predecían la facilidad con que los sujetos las recordaban cuando se les pedía una lista de emociones, o la probabilidad de que las denominaran emoción cuando se les preguntaba qué eran, o la facilidad con la que podían ser sustituidas por la palabra emoción en una frase sin que ésta perdiese sentido, el grado en que se parecían a otras categorías intermedias de emoción en base a atributos compartidos y la velocidad con la que los sujetos podían verificar que eran en verdad una emoción. En síntesis, diferentes evidencias convergentes muestran que el concepto de emoción tiene una estructura interna y que dicha estructura predice varios índices de procesamiento cognitivo que implican conceptos de emoción.

Además, los sujetos de estos estudios no lograron enumerar los atributos necesarios y suficientes de emoción. Cuando se les preguntó, enumeraban atributos tales como un aumento de los latidos cardiacos, de la transpiración, lágrimas, ojos muy abiertos y una preocupación obsesiva por la situación -atributos que no son ni necesarios ni suficientes-. Hay casos claros de emociones prototípicas, tales como la felicidad y la tristeza, que carecen de todos esos atributos.

También de acuerdo con la hipótesis, se encontró que el concepto emoción tenía fronteras difusas. A los sujetos se les hicieron preguntas como: « $i$ Es $\mathrm{X}$ un tipo de emoción?», siendo $\mathrm{X}$ reemplazada por varias palabras que denotan 
tipos de emoción (Fehr y Russell, 1984). Según la visión clásica, ésta debería ser la más fácil de las preguntas. La pregunta sería simplemente si el conjunto de atributos definitorios de, por ejemplo, orgullo incluyen el conjunto de atributos que definen emoción. Si una persona, ya sea implícita o explícitamente, conoce los atributos necesarios y suficientes para orgullo y emoción, no debería ser ambigua la tarea de decidir cuando el orgullo es una emoción -igual que no debería haber ambigüedad para nadie que conozca el significado de las palabras padre y madre, al decidir si una madre es o no un tipo de progenitor-. Pero, de hecho, los sujetos no podían decidir si el orgullo es una emoción. Algunos decían que sí, otros decían que no. En varios estudios aún no publicados, también hemos encontrado fronteras difusas para amor (Fehr y Russell, 1989) y para enojo (Russell y Fehr, 1989).

Estos resultados para amor, enojo y emoción se refieren a la definición de los conceptos. En otra serie de estudios examinamos cómo los adultos usan los conceptos del nivel básico para categorizar el mensaje transmitido por expresiones faciales emocionales (Russell y Bullock, 1986). Como se predijo, las expresiones faciales variaban en su grado de ejemplaridad. Algunas expresiones eran ejemplos prototípicos, otras eran ejemplos intermedios y otras, aún, eran muy malos ejemplos. Había también casos fronterizos en los cuales los sujetos no podían decidir si una expresión facial concreta era o no miembro de una categoría emocional específica.

\section{Argumentos y réplicas}

Más que proseguir con un análisis de los prototipos, voy a centrarme en la cuestión de si esta perspectiva es la correcta. La defensa de la alternativa clásica puede ser presentada con cinco afirmaciones (no quiero decir con ello que todos los críticos de la visión prototípica acepten estas cinco afirmaciones):

\section{El concepto supraordenado de emoción está definido clásicamente}

Johnson-Laird y Oatley (1987) proporcionaron una definición clásica de emoción, pero Ortony et al. (1987) se limitan a afirmar que el fracaso desde el punto de vista clásico para producir una definición aceptable de emoción «no demuestra que este objetivo sea imposible» (p. 334).

Consideremos en primer lugar la afirmación de Ortony et al. No creo que una definición clásica sea en términos lógicos una contradicción en sí misma. Ningún argumento ni evidencia podrían, pues, establecer la imposibilidad de una definición clásica. La cuestión es más bien otra de carácter empírico: si el concepto cotidiano de emoción es de hecho definido clásicamente. Los defensores del punto de vista clásico tienen a su disposición una manera obvia de silenciar cualquier actitud escéptica sobre este asunto: producir una definición clásica para emoción y demostrar que la definición propuesta es la que la gente conoce, cuando conoce el significado del término. (No es necesario que la gente sepa conscientemente cuáles son los atributos necesarios y suficientes, pero deberían comportarse como si estos atributos constituyeran el significado de emoción). Para que sea más que una posibilidad abstracta, la posición clásica necesita producir una definición específica y aceptable de la emoción. Examinemos algunos posibles candidatos.

Las teorías filosóficas de la emoción han intentado, a menudo, establecer cuáles son las condiciones necesarias y suficientes para que algo sea una emo- 
ción. Lyons (1980) toma explícitamente esta condición como un criterio de éxito para una teoría de la emoción. Aunque Lyons no está preocupado por el significado que el hablar cotidiano da a la emoción, su análisis demuestra que incluso los expertos han fracasado en proveer una definición clásica satisfactoria. Todas las aproximaciones a la emoción consideradas - desde Aristóteles hasta Skinner - resultaron insatisfactorias. En otras palabras, de acuerdo con Lyons (1980), nadie ha planteado aún una definición clásica adecuada para emoción.

Lyons, entonces, ofrece una propia: «Algo puede ser denominado como un estado emocional si y sólo es un estado fisiológico anormal provocado por la evaluación que el sujeto de ese estado hace de su situación» (pp. 57-58) (2). Si Lyons está en lo cierto, entonces la teoría de Izard (1977) que plantea que todos estamos siempre en un estado emocional, significaría que todos estamos siempre en un estado fisiológico anormal. Considerando cualquier definición razonable de anormal, Izard no sólo estaría equivocado, sino que estaría equivocado por definición. Si Lyons tiene razón, entonces la hipótesis de que la felicidad puede ocurrir sin ningún cambio fisiológico es una contradicción. Si Lyons está en la posición correcta, entonces todas las emociones deben ser provocadas por una evaluación. Una emoción no podría ser, por definición, inducida química o fisiológicamente de una manera tal que no pasara por una evaluación. La tesis de Zajonc (1980), de que la emoción precede a la cognición, estaría equivocada por definición. Si Lyons tiene razón, entonces, por definición, cualquier estado (incluyendo las enfermedades psicosomáticas, la indigestión, el deseo, el hambre y el dolor) es una emoción si existe un estado fisiológico anormal provocado por la evaluación de la situación que hace el sujeto de ese estado.

Ortony et al. (1987) examinaron aproximadamente quinientas palabras asociadas a la emoción, buscando aislar las que se refieren a la emoción. Su conclusión fue la siguiente:

«Los mejores ejemplos de los términos emocionales demuestran ser aquellos que (a) se refieren a condiciones internas, metales, como contrapuestas a las físicas o externas, (b) son claros ejemplos de estados y (c) consideran, como un punto de referencia central (no accesorio), que el afecto es algo opuesto a la conducta o la cognición. Ignorar una de estas condiciones conlleva dar peores ejemplos o en absoluto ejemplos de emociones; sin embargo, esta gradación no la tomamos como evidencia de que las emociones se resistan necesariamente a cualquier definición clásica» (p. 341).

Ortony y otros (1987) no intentan establecer una definición clásica de emoción, ni tampoco que su análisis proporcione una única categoría que contenga a todos y sólo a aquellos términos que denotan emociones. Sin embargo, ellos encontraron diversos grados de pertenencia. Puede argumentarse que su análisis pueden llevar a una definición clásica, pero soy escéptico frente a esa posibilidad, en parte porque dudo de que se pueda progresar mucho definiendo emoción en términos de afecto tal como hacen Ortony et al. y, en parte también, porque dudo de que pueda demostrarse que la definición resultante es relevante en los procesos psicológicos que median en el uso del lenguaje.

Johnson-Laird y Oatley (1986) ofrecen la siguiente definición de emoción: «El concepto ordinario de emoción no es, pues, más que un "contenedor" para un conjunto disjunto de estados mentales -emociones específicas- que contrastan con otros tipos de cogniciones» (p.36).

Específicamente, la emoción es felicidad, tristeza, miedo, enojo o asco. Estas cinco son, a su vez, primitivos semánticos que no tienen atributos semánticos. 
Johnson-Laird y Oatley (1986) tienen sus propias razones teóricas para agrupar felicidad, tristeza, enojo, miedo y asco en una categoría. Pero no explican por qué la gente tendría que hacerlo así. Es decir, si felicidad, tristeza, enojo, miedo y asco carecen de atributos semánticos, no está claro en base a qué los sujetos, al hablar cotidianamente, las agruparían, ignorando otros estado psicológicos para formar la categoría emoción.

Su definición de emoción carece también de una explicación para los resultados resumidos anteriormente (Fehr y Russell, 1984, 1989). Por ejemplo, ¿por qué serían algunos miembros de este conjunto juzgados como mejores ejemplos de emoción que otros? Parece ser especialmente dificultoso para Johnson-Laird y Oatley explicar por qué los sujetos pueden de manera fiable juzgar que amor -que no está en la lista de los cinco- es un mejor ejemplo de emoción que asco - que sí está en dicha lista-. Si la definición de Johnson-Laird y Oatley es correcta, la mayoría de los sujetos a los que se les pider que hicieran una lista de emociones deberían enumerar rápidamente estas cinco, de hecho, en el estudio de Fehr y Russell (1984) solo el 14\% de los sujetos mencionaron amor, odio, ansiedad y depresion. Según la hipótesis de Johnson-Laird y Oatley, decir que el asco no es una emoción sería generar una contradicción. Sin embargo, el 6\% de los sujetos de Ferh y Russell (1984) y el $26 \%$ de los sujetos de Shield (1984) negaron que el asco sea una emoción. Al presentar su teoría de la emoción, Pankseep (1982) da diversas razones que cuestionan que el asco sea una emoción. El problema no es cuándo el asco es o no es una emoción: la pregunta es cómo cualquiera - ya sea un lego o un experto- puede llegar a tal conclusión si el asco es, por definición, una emoción. Sería lo mismo que negar que una madre es un pariente, es decir, lo mismo que no saber el significado de las palabras que utilizamos.

En síntesis, la definición clásica de emoción es aún una posibilidad abstracta, pero no he logrado ver avances hacia la obtención de una definición de este tipo. Incluso los que aceptan el punto de vista clásico no parecen converger hacia una candidatura única. De las tres candidaturas que hemos considerado, sólo una fue propuesta por sus autores como una definición clásica para el concepto de emoción del lenguaje natural, y las tres difieren sustacialmente entre sí. Johnson-Laird y Oatley (1986), argumentan que emoción se define en términos de miedo, enojo, felicidad, tristeza y asco, los cuales, a su vez, no tienen atributos definitorios. Lyons (1980) argumenta que la definición de estado emocional incluye como atributo necesario la referencia a un estado fisiológico, mientras que Ortony et al. (1987) dicen que los términos emocionales se refieren a estados mentales que se oponen a estados físicos. Como se puede ver, no hay acuerdo sobre una definición clásica de emoción.

2. Los conceptos emocionales de nivel básico están definidos clásicamente.

Ortony et al. (1987), defienden la aproximación clásica para los términos emocionales de nivel básico, apoyándose en que Wierzbicka $(1972,1973)$ ha proporcionado definiciones de estos términos. Sin embargo, las definiciones de Wierzbicka no eran definiciones clásicas. Wierzbicka (1972) argumenta contra la existencia de atributos necesarios y suficientes para definir los términos emocionales. Aún más, en otro contexto, Wierzbicka (1984, p. 213) escribe: «En el caso de las palabras que describen clases naturales o clases de artefactos humanos, el comprender la estructura de los conceptos significa describir completa y exactamente la idea (no sólo la imagen visual) de un representante típico de la clase, este es, el prototipo.» 
Wierzbicka (1973) ofrece un esquema general para la definición de los conceptos de emoción:

«Este esquema general es una comparación: una comparación entre una emoción sentida por la persona de la que se habla, y lo que la memoria o la imaginación evocan en nosotros en relación con la descripción de la situación, un movimiento del cuerpo o una expresión facial, o una reacción fisiológica» (p. 505).

El esquema general de Wierzbicka es otra manera de plantear la presente hipótesis: un acontecimiento es juzgado como una emoción concreta comparándolo con un script archivado en la memoria. El script incluye la situación, los movimientos corporales, expresiones y reacciones fisiológicas típicas de esa emoción.

Johnson-Laird y Oatley (1989) argumentan que miedo, enojo, felicidad, tristeza y asco son primitivos semánticos $\mathrm{y}$, por tanto, no pueden ser definidos. (Clore y Ortony estarían en desacuerdo con esta idea, como cualquiera que dude de que estos conceptos sean panculturales, como por ejemplo, Lutz, 1989; Howell, 1897; Wierzbicka, 1987.) Para probarlo, Johnson-Laird y Oatley señalan que la siguiente afirmación es aceptable: «Juan se siente enojado, pero no sabe por qué.» $\mathrm{Si}$ tal afirmación es aceptable, entonces conocer el porqué no es un atributo necesario del enojo. En la hipótesis del script, esta afirmación también es aceptable porque ningún atributo del script es necesario. La mencionada afirmación podría ser mucho más larga: «Juan se siente enojado pero no sabe por qué; no cree que lo hayan agredido u ofendido; no percibe injusticias; no se siente tenso, ni agitado, ni acalorado. No está mirando mal a nadie, ni tiene el ceño fruncido; su pulso no ha cambiado; no siente rígidos sus músculos; no está pidiendo una reparación; no está perdiendo el control, en realidad no tiene impulsos agresivos que controlar, no ha gritado ni ha agredido a nadie.» Desde el punto de vista de la aproximación de Johnson-Laird y Oatley esta afirmación es aceptable porque el enojo no tiene atributos definitorios. Sin embargo, desde el punto de vista del script, esto no es aceptable. Yo quisiera preguntarle a la persona que dijera tal frase: «¿Que es, entonces, lo que quiere usted decir cuando afirma que Juan está enojado?.»

Otras palabras emocionales de nivel básico son definidas por Johnson-Laird y Oatley en términos de las cinco más básicas. Por ejemplo, amor es definido de la siguiente manera: Amar es «tener la experiencia de una felicidad interna en relación a un objeto o a una persona, que también puede ser objeto de deseo sexual» (p. 116). El mandamiento «Ama a tu prójimo» pierde algo importante cuando se traduce como «Experimenta felicidad interna en las relaciones con tu prójimo». Desde la perspectiva de los prototipos, la felicidad puede ser sólo uno de los muchos atributos que caracterizan al amor; y, quizás, no el más característico, además. Nuestros sujetos no enumeran la felicidad como uno de los atributos de la mayoría de los tipos de amor (Fehr y Russell, 1989). Las características compartidas por la mayoría, sino por todos los tipos de amor, eran: cuidado, ayuda, establecer un lazo, compartir, sentirse libre para hablar de lo que uno quiere, comprensión, respeto y cercanía. La definición de Johnson-Laird y Oatley implica que si se experimenta felicidad interna en relación a un objeto o persona, entonces, por definición, se está experimentando amor. Dicho de otro modo, una relación no amorosa pero feliz sería una contradicción en términos. Su definición también implica que si se está experimentando amor, entonces se debe estar experimentando felicidad. En otras palabras, una relación amorosa infeliz debería ser una contradicción en términos. 
Las distintas propuestas consideradas aquí no cuadran las unas con las otras, ni tampoco hay evidencia empírica que establezca una definición clásica para ninguno de los términos de nivel básico. De hecho, aunque Johnson-Laird y Oatley (1989) argumentan que la mayoría de las palabras emocionales pueden ser definidas clásicamente, ellos rechazan la definición clásica para los cinco conceptos emocionales claves. Así pues, se puede decir que no hay un acuerdo sobre la definición clásica para los términos de nivel básico, aun entre aquellos que aceptan el punto de vista clásico.

\section{La estructura interna no contradice el punto de vista clásico.}

La mayoría de la evidencia reunida en la perspectiva de los prototipos se orientó a establecer que los miembros varían en su grado de pertenencia. La pertenencia gradual es denominada estructura interna del concepto en cuestión. Tanto Johnson-Laird y Oatley $(1986,1989)$ como Ortony et al. (1987) argumentan que la estructura interna puede darse de hecho en los conceptos emocionales, incluso en la mayoría de los conceptos, pero que esto no contradice el punto de vista clásico. Armstrong, Gleitman y Gleitman (1983) muestran que conceptos definidọs clásicamente como «número impar» y «figura geométrica plana» tienen una estructura interna. Los sujetos, por ejemplo, eligen el 3 y el 7 como mejores ejemplo de número impar que 15 o 427. Dado que número impar y figura geométrica plana son casos paradigmáticos de conceptos definibles clásicamente, esta evidencia muestra claramente que una estructura interna y una definición clásica no son posibilidades mutuamente excluyentes.

Los argumentos de Johnson-Laird y Oatley, así como los de Ortony et al. están equivocados al atacar una posición que, en realidad, nadie defiende. Lejos de reclamar que la estructura interna contradice el punto de vista clásico, Fehr y Russell (1984) afirmaban: «La existencia de una estructura interna no contradice el punto de vista clásico de los conceptos. Demostrar que un concepto tiene estructura interna no significa que no posea fronteras rígidas o criterios definitorios de pertenencia» (p. 474).

En primer lugar, en este caso el problema es empírico: la existencia de una estructura interna erosiona el punto de vista clásico basándose en la descripción de cómo una persona puede usar y comprender un concepto sin conocer sus atributos necesarios y suficientes. El punto de vista clásico no predijo y, sin afirmaciones ad hoc, no puede dar cuenta de la estructura interna. La estructura interna es un atributo significativo de conceptos emocionales, de hecho de la mayorías de ellos, prediciendo aspectos del procesamiento cognitivo de los miembros de una categoría tales como tiempo de reacción, accesibilidad e inferencias inductivas. Fue la perspectiva de los prototipos la que reveló estas relaciones empíricas.

Segundo, Fehr y Russell (1984) distinguieron la estructura interna de las fronteras difusas y plantearon que era la existencia de tales fronteras lo que contradecía el punto de vista clásico. Así, la evidencia empírica más llamativa sobre la no definición clásica de un concepto sería el hecho de que la gente no puede diferenciar a los miembros de los no miembros. La existencia de tales casos ambiguos fue presentada como la evidencia fundamental de nuestra argumentación contra la aproximación clásica al concepto supraordenado de emoción (Fehr y Russell, 1984), a los conceptos de nivel básico de enojo (Russell y Fehr, 1989) y amor (Fehr y Russell, 1989) y a nuestro análisis de la categorización de las 
expresiones faciales de emoción (Russell y Bullock, 1986). La evidencia empírica sobre estos casos ambiguos es el tema de la próxima sección.

\section{La evidencia de los casos ambiguos no contradice el punto de vista clásico.}

Los resultados que demuestran que los sujetos no pueden decidir qué casos son, y cuáles no, miembros de una categoría han sido citados anteriormente como la evidencia más fuerte de que las categorías no han sido definidas clásicamente. Los críticos al punto de vista prototípico han planteado varias contraargumentaciones a esta evidencia.

Smith y Medin. Smith y Medin (1981) sugieren dos maneras para reconciliar el punto de vista clásico con la evidencia empírica sobre casos ambiguos. Quizás - señalan - una palabra etiqueta dos conceptos: un concepto del lenguaje ordinario y un concepto técnico (por ejemplo, «fruta» es simultáneamente un concepto cotidiano y un concepto biológico). Los sujetos no pueden decir si el tomate es una fruta porque ellos no pueden decidir si la palabra fruta está siendo utilizada en un sentido cotidiano o en un sentido técnico. Este argumento, sin embargo, tiene poco que aportar en el dominio de las emociones, ya que orgullo, enojo y emoción carecen de sentidos técnicos.

La segunda posibilidad que ellos plantean es que los sujetos no dominan el concepto, sino que poseen una versión incompleta o limitada. Aun si esto preservara el punto de vista clásico, este viraje convertiría la perspectiva en una explicación irrelevante para los conceptos de muchos hablantes. Rosch (1977) estudió lo que los sujetos universitarios, cuya lengua materna era el inglés, quieren decir con palabras cotidianas tales como vehículo (vebicle), vegetal (vegetable) y deporte (sport). Mis colegas y yo hemos examinado lo que se entiende por emoción, amor, enojo y otras palabras parecidas. La evidencia indica que esta gente, que sin duda conoce el significado de estas palabras, no conocen sin embargo, ni siquiera tácitamente, los atributos clásicamente definitorios para estas palabras. Si esto es así, los atributos definitorios clásicos no constituyen el significado de estas palabras.

(Los planteamientos de Smith y Medin ponen de relieve la ambigüedad de la cuestión sobre si el punto de vista clásico debe ser interpretado como algo concerniente a los conceptos cotidianos o a los conceptos técnicos. En esta parte del artículo, mi tema es la aproximación clásica a los conceptos cotidianos y, por tanto, criticaré sus planteamientos, pero podría estar de acuerdo con una versión ligeramente modificada de la idea que subyace a los dos planteamientos de Smith y Medin. La aproximación de los prototipos da cuenta de cómo la gente corriente comprende los conceptos cotidianos. Las definiciones clásicas son algo logrado por expertos con propósitos técnicos. La gente corriente, sin embargo, a veces toma prestadas etiquetas de conceptos técnicos sin una comprensión total de su significado. Los expertos, algunas veces, toman prestadas etiquetas cotidianas para sus conceptos técnicos. Los conceptos técnicos surgen, a veces, a partir de los conceptos cotidianos.)

Johnson-Laird y Oatley. Johnson-Laird y Oatley (1986) también sugieren dos posibilidades. Primero, que los argumentos basados en casos ambiguos confunden el significado con la identificación. Una abuela puede ser identificada por su pelo gris, las arrugas alrededor de los ojos y su empeño en que tomemos sopa de pollo, pero la palabra «abuela» significa madre-de-un-padre/madre. La evi- 
dencia obtenida respecto a casos ambiguos muestra que lo que está mal definida es la función de identificación, no el significado.

Esta refutación puede ser relevante para la evidencia de Russell y Bullock (1986) referente a cómo la gente categoriza las expresiones faciales de emoción, pero es irrelevante para los resultados de Fehr y Russell (1984) sobre casos ambiguos. A los sujetos se les pedía contestar si o no a preguntas del tipo: «¿Es $\mathrm{X}$ una emoción?». Su incapacidad para lograr una respuesta única hace referencia al significado (intensión) de sus conceptos y no tiene nada que ver con la función de identificación. Decidir si, por ejemplo, orgullo es una emoción no es identificar personas orgullosas; más bien es realizar la tarea semántica de decidir si un ejemplo de orgullo es o no, por definición, un ejemplo de emoción. No necesitamos ser capaces de identificar cuadrados concretos para saber que un cuadro es un rectángulo, ni identificar serafines y querubines para saber que son angeles.

En segundo lugar, Johnson-Laird y Oatley argumentan que palabras tales como orgullo son homónimas. Etiquetan dos conceptos y mis sujetos podrían haber logrado dar una respuesta única si el contexto hubiera permitido aclarar a qué concepto se hacía referencia. Esta idea es semejante a la de Smith y Medin, pero aduciendo que las palabras tienen diferentes sentidos cotidianos. Para ilustrarla, Johnson-Laird y Oatley señalan que:

«El sustantivo "orgullo" es mencionado en la mayoría de los diccionarios como poseedor de varios significados diferentes, incluyendo: 1 . Sentido de nuestra propia dignidad o valor. 2. Placer o satisfacción que se obtiene a partir del propio trabajo, logros o posesiones» (pp. 34-35).

Es de suponer que, si a los sujetos se les dijera a cuál de estos significados nos estamos refiriendo, entonces podrían estar de acuerdo sobre si orgullo es o no una emoción.

En este razonamiento, Johnson-Laird y Oatley expresan un constante optimismo respecto a que las definiciones clásicas son rápidamente accesibles y que las dificultades semánticas que los sujetos tienen en relación a estas palabras son fácilmente superables. Para contrastar esta idea en particular, pregunté a un número de sujetos: «¿Es orgullo (palabra que significa sentido de nuestra propia dignidad o valor) una emoción?.» $\mathrm{A}$ otros sujetos les pregunté: «¿Es el orgullo (palabra que significa satisfacción o placer que se obtiene a partir del propio trabajo, logros o posesiones) una emoción?.» Como se puede ver en los resultados en la Tabla 2, los sujetos no lograron, de nuevo, ponerse de acuerdo.

TABLA 2

¿Es ongullo una emoción?

Definición

SI

NO

satisfacción o placer que

se siente por el trabajo,

logros o posesiones de uno

47

14

sentido de la propia

dignidad o valor

36

20

NOTA:

Los sujetos fueron estudiantes universitarios que respondieron a un breve cuestionario durante una clase. Los sujetos fueron asignados a una condición aleatoriamente. $\chi^{2}(1 ; N=117)=2.32$, n.s. 
Creo que los sujetos siguieron teniendo dificultades porque orgullo no es un homónimo (dejando aparte la rara acepción de pride, en inglés, como «grupo de leones»). Los dos «significados» enumerados por Johnson-Laird y Oatley como homónimos están demasiado íntimamente relacionados para que sea una coincidencia el que se les etiquete con la misma palabra (considérese cuán distintos son otros homónimos, tales como banco: institución financiera y asiento de madera). El prototipo de orgullo consta tanto de un sentido del propio valor como del de la satisfacción que conlleva.

Desde una perspectiva más general, el planteamiento de Johnson-Laird y Oatley respecto a los homónimos tiene que hacer frente a dos problemas. En primer lugar, muchos términos emocionales de nivel básico deberían ser homónimos. Orgullo no es el único caso ambiguo; la mayor parte de los ejemplares no prototípicos provoca una falta de acuerdo entre los sujetos (Fehr y Russell, 1984: Shields, 1984). Así pues, Johnson-Laird y Oatley tendrían que convencernos de que todas estas palabras son homónimos.

En segundo lugar, Johnson-Laird y Oatley deberían haber ideado un método para comprobar su idea que no fuera su determinación a través del contexto. Aun cuando el contexto no aclaró el significado de orgullo en este estudio, no dudo de que el contexto pueda aclarar el significado asignado a cualquier palabra. El problema es que una demostración de este efecto no favorece la perspectiva clásica en favor de la de prototipos, ya que el contexto puede aclarar tanto un concepto borroso como un homónimo. En el contexto de la frase «hay muchas horas en un día», puede asumirse que muchas adquiere el significado preciso de 24. La cuestión es que, en sí misma, muchas no tienen ese significado preciso.

Ortony, Clore y Foss. Ortony et al. (1987) replantean el problema de la siguiente manera: para poder decidir cuándo $\mathrm{X}$ es miembro de una categoría $\mathrm{Y}$ se requieren al menos dos informaciones: (1) las condiciones necesarias y suficientes para pertenecer a la categorías $Y(2)$ si $X$ tiene las propiedades relevantes. Así pues, dicen, el fracaso en decidir la pertenencia a una categoría «puede también considerarse que establece grados diferentes de ignorancia» (p. 345). Para ilustrar este punto, Ortony et al. imaginan preguntar a los sujetos si 356.489.132.017 es o no un número primo. El fracaso de los sujetos en ponerse de acuerdo no significa que la categoría «número primo» sea una categoría difusa. «Podría sencillamente probar que los sujetos no saben (y no pueden resolverlo en un tiempo razonable) si el cándidato tiene o no las propiedades para su inclusión en el conjunto de números primos.» (p. 5).

Decidir cuándo el orgullo es o no una emoción requiere, según Ortony et al. (1987), conocer dos informaciones diferentes: (1) Las condiciones necesarias y suficientes para ser miembro de la categoría emoción y (2) si orgullo tiene las propiedades relevantes. El fracaso al decidir que un elemento pertenece a la categoría podría indicar la ignorancia de la condición (1) de (2) o de ambos. La ignorancia en el primer caso (1) podría ser de los atributos que definen emoción. Esto es lo que se quiere decir cuando se afirma que alguien no conoce la definición clásica de emoción. La ignorancia en el segundo caso (2) podría ser de los atributos que definen orgullo. Esto es lo que se quiere decir cuando se afirma que la gente no conoce la definición clásica de orgullo. En cualquier caso, el punto de vista clásico fracasa, aunque la evidencia no señale específicamente en cuál de los dos términos. 
Consideremos ahora el ejemplo de Ortony et al. (1987), en relación a los números primos. Ser número primo (o no serlo, como podría ser el caso) está determinado por la definición, pero no es parte de la definición de 356.489.132.017; de modo que es preciso realizar cálculos. Número primo es un concepto técnico, no es un concepto cotidiano. Sabemos que la categoría número primo está definida clásicamente, porque los que conocen el significado de la frase pueden especificar sus atributos necesarios y suficientes. Además, 356.489.132.017 es o no es un número primo. Todos los expertos, si se les da la oportunidad de trabajar en el problema pueden llegar a un acuerdo en la respuesta. (De hecho, dando suficiente tiempo e incentivo, cualquiera que conozca el significado de número primo debería llegar a la respuesta correcta.) No veo ninguna razón para suponer que este sea el caso para emoción. Aunque los filósofos y los psicólogos lo han intentado durante siglos, nadie ha enumerado los atributos emoción que sean aceptados comúnmente como necesarios y suficientes. Un indicador muy importante en este problema es que los expertos están en desacuerdo sobre los casos específicos. Aunque Hume (1739-40/1978) analizó el orgullo como un caso paradigmático de emoción, algunos de mis colegas han negado que el orgullo sea una emoción. Ekman, Friesen y Simons (1985)

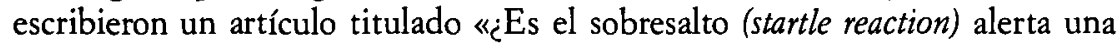
emoción?», una pregunta sobre la cual, como ellos dicen, «los teóricos de la emoción han estado en desacuerdo» (p. 1.416). Después de argumentar que el sobresalto no es una emoción, reconocen que «S. S. Tomkins no apreciaría nuestros resultados como un cuestionamiento a su afirmación que el sobresalto es una emoción» (p. 1.424). El amor fue señalado por los sujetos como uno de los ejemplos más prototípicos de emoción (Fehr y Russell, 1984) y, sin embargo, hay colegas que niegan que el amor sea una emoción y, en su lista de emociones muy a menudo no mencionan el amor. Las mismas listas de emociones muy a menudo incluyen sorpresa, culpa e interés y, sin embargo, Clore y Ortony (1988) plantean sus sospechas de que éstas, «lejos de ser emociones básicas, pueden no ser ni siquiera emociones» (p. 368). Entonces, decidir si amor, orgullo, sobresalto, sorpresa, interés y culpa son emociones, no puede hacerse por el significado de los términos emoción, orgullo, sobresalto, etc., aun cuando nos apoyemos en los significados que los expertos dan a estos términos.

\section{Las definiciones clásicas para emoción, enojo, etc., podrán ser descubiertas cien-} tificamente algún día.

Aunque actualmente la gente que habla el lenguaje natural no pueda dar definiciones clásicas para términos como emoción, enojo, amor y otros parecidos, ¿no podrían descubrirse sus atributos necesarios y suficientes a partir de la investigación científica sobre casos concretos de emoción? Clore y Ortony (1988) mantienen la esperanza de que algún día se puedan descubrir algunos atributos comunes en los hechos emocionales mismos. Creo que esto es posible, pero muy poco probable.

Primero, hay que darse cuenta de que esto es un abandono parcial del punto de vista clásico que estamos considerando aquí, que plantea que las características definitorias existen en los hechos categorizados (la extensión del concepto) y que también son tácitamente conocidas por cualquiera que conoce el significado del nombre de la categoría (intensión) -el conjunto de características que constituyen el significado del nombre de la categoría-. La desconexión de es- 
tos dos elementos convierte en una coincidencia el descubrimiento de las características definitorias: una categoría de hechos (por ejemplo, las emociones) seleccionadas de acuerdo con un conjunto de criterios (tales como el parecido con una familia de prototipos) debería poseer, sin saberlo el que ha hecho la selección, otro conjunto de características comunes a todos los casos y únicas como conjunto para esta categoría. No hay razones para suponer una cosa así una vez abandonada la idea de que los hablantes utilizan las características definitorias para seleccionar los casos.

Más aún, es poco claro qué acontecimientos está investigando el científico porque todavía no hemos podido ponernos de acuerdo sobre qué hechos son o no son emociones. ¿Podrían los científicos investigar lo que es orgullo o el sobresalto? Clore y Ortony (1968) sugirieron que podemos concentarnos en los casos claros, los prototipos, y dejar los casos fronterizos para asignarles posteriormente sus categorías. (Encontrar primero los atributos necesarios y suficientes del enojo, del miedo y de otros casos claros. Si el orgullo tiene esos atributos, entonces es una emoción: si no no lo es.) Sin embargo, creo que a esta postura se le pueden aplicar los mismos elementos críticos anteriores. Nadie ha postulado atributos necesarios y suficientes ni siquiera para las emociones prototípicas y debería haber acuerdo si existieran. Lo que es más, la prototipicidad es graduada. No podemos ponernos de acuerdo en un caso claramente fronterizo de emociones prototípicas y emociones no tan prototípicas. No sabemos si el científico debería incluir o no el amor, el asco, el interés, la culpa y la sorpresa. El enojo y el miedo son conceptos difusos, incluso aunque todos los científicos se pusieran de acuerdo en que, por ejemplo, el miedo y el enojo son al menos casos claros de emociones. Por tanto, es poco claro qué casos específicos de enojo y miedo debería investigar el científico.

$\mathrm{Al}$ margen de estos problemas, supongamos que el investigador encuentra algunos atributos comunes a todos los casos claros de miedo y de enojo (por ejemplo, niveles anormales de hormonas específicas e incremento de actividad de ciertos nervios). ¿Podrían entonces, estos atributos comunes definir emoción? Estos atributos podrían no existir en la mayoría de los acontecimientos que la gente considera emociones. Estos atributos podrían no ser aquellos a partir de los cuales el común de la gente que habla un mismo lenguaje utiliza para la palabra emoción. Estos atributos podrían no explicar ni predecir inferencias inductivas, la accesibilidad a la memoria o los juicios de hablantes competentes sobre las emociones. Sin embargo, los atributos descubiertos a los que hemos hecho referencia podrían ser útiles y crear nuevos conceptos técnicos para la psicología de la emoción. Este es el tema que analizaremos en la sección siguiente.

\section{Hacia una reconciliación de puntos de vista}

Queda la posibilidad de que el punto de vista clásico pueda ser construido de otras maneras. Los defensores del punto de vista clásico podrían haber estado hablando acerca de cómo los psicólogos podrían conceptualizar de mejor forma las emociones. Podrían haber estado motivados por la creencia de que los conceptos deberian ser definidos clásicamente. Pueden haber creído que necesitaban defender el punto de vista clásico (y criticar el punto de vista de los prototipos) con vistas a justificar la creación de conceptos definidos clásicamente para el uso científico. Si esto fuera así, el desacuerdo podría haber surgido de un malentendido y de un intercambio de monólogos. Quisiera sugerir aquí una 
posible reconciliación, diferenciando entre los usos descriptivos y prescriptivos de una definición clásica.

Llamemos uso descriptivo de una definición clásica a la aproximación empírica a la forma en que los hablantes ordinarios entienden cotidianamente la palabra que ellos usan. En esta acepción, la definición clásica debería tener una realidad psicológica. Esta es la acepción de definición clásica presupuesta en la de los comentarios anteriores.

Llamemos prescriptiva a la utilización de la definición clásica como una meta a lograr por los expertos. El concepto definido clásicamente debería ser creado a través de un análisis científico de los hechos conceptualizados. Según esta interpretación, Johnson-Laird y Oatley deberían estar diciendo algo así como: nosotros, los psicólogos, haríamos bien en definir para usos científicos una categoría claramente delimitada que constara de un conjunto disjunto de cinco estados cerebrales distintos (a determinar empíricamente por medio de datos fisiológicos panculturales). Ortony y Clore podrían decir que los psicólogos haríamos bien definiendo para usos científicos una categoría claramente delimitada de estados mentales internos cuyo punto de referencia es el afecto. Lyons podría estar diciendo que hariamos bien en definir una categoría de estados fisiológicos anormales causados de cierto modo.

Nada de lo que he dicho en este artículo está en desacuerdo con los objetivos planteados de esta manera. Nadie está criticando que puedan crearse conceptos definidos clásicamente. Planteado de esta manera, sus objetivos no están en desacuerdo con ninguno de los análisis de los conceptos del sentido común ni hay desacuerdo entre unos y otros. Los propósitos prescriptivos son cursos de acción que han de ser evaluados por su utilidad para la ciencia. No son evaluados en términos de si coinciden o no coinciden con la psicología ingenua o con los significados cotidianos de las palabras.

No sé si estos autores querrían restringirse ellos mismos a una interpretación prescriptiva. Algunos pasajes de sus textos sugieren interpretaciones prescriptivas de definiciones clásicas. (3) Por otro lado, la aceptabilidad de las frases, el análisis del léxico emocional y los test lingüísticos sobre el significado de las palabras no son criterios de referencia a partir de los cuales evaluar los análisis prescriptivos de los acontecimientos a los que se refieren las palabras emocionales.

Los científicos pueden definir nuevos conceptos de cualquier manera que consideren necesaria y les pueden asignar la etiqueta que deseen. Sin embargo, el cómo estos conceptos sean etiquetados no debe determinar su relación con los conceptos utilizados actualmente. De cualquier forma que se etiqueten, los conceptos definidos por atributos necesarios y suficientes no pueden ser los conceptos que se expresan cotidianamente con las palabras emoción, enojo y otras similares. Si los conceptos son hoy difusos y mañana están más claramente definidos, entonces no son los mismos. Los nuevos conceptos no definen a los viejos que ya nos son familiares. Es equívoco entonces pensar que emoción (o enojo o cualquiera de los otros términos), tal como lo entendemos en la actualidad, podrá ser algún día definido clásicamente. La etiqueta asignada a un concepto claramente delimitado es irrelevante, pero podemos imaginar la confusión que resultaría de utilizar etiquetas tales como emoción, enojo, etc. Es probable que ni la intensión ni la extensión de estos nuevos conceptos coincida con la intensión o extensión de los conceptos cotidianos etiquetados actualmente como emoción, enoio, etcétera. 


\section{Resumen}

No sé si los autores que proponen un punto de vista clásico para los conceptos emocionales están planteando el uso de una definición clásica en un sentido descriptivo o prescriptivo. Si el uso que ellos plantean es descriptivo (o simultáneamente descriptivo y prescriptivo), continuaríamos estando en desacuerdo; sin embargo, si es prescriptivo, el desacuerdo puede desaparecer.

En este artículo he argumentado que el uso descriptivo de las definiciones clásicas para palabras inglesas como emotion, anger, love (emoción, enojo, amor), etc, no han logrado explicar lo que cotidianamente entendemos por medio de esas palabras. La aproximación clásica a los conceptos no ha producido definiciones viables, no ha logrado predecir la conducta de los sujetos y no ha proporcionado una explicación clara de los resultados empíricos disponibles en la actualidad. No creo que una definición clásica sea una contradicción lógica en sí misma y no he tratado de establecer la imposibilidad de la aproximación clásica. Mi crítica es empírica. El punto de vista clásico es una hipótesis legítima, pero el punto de vista prototípico da cuenta, en una forma mucho más prometedora, de la evidencia empírica disponible. Señalar que es posible crear una definición clásica en el estudio de las emociones es irrelevante para la hipótesis de que los conceptos ordinarios de las emociones son prototípicos más que organizados clásicamente. La tarea próxima es explorar alguna de la variantes de las alternativas no clásicas. Por ejemplo, Conway (en prensa) y Conway y Bekerian (1987) argumentan convincentemente sobre una representación heterogénea y compleja de los conceptos emocionales que incluye scripts, memorias autobiográficas, escenas prototípicas y varias proposiciones transmitidas culturalmente.

El tema del análisis prototípico es el de los conceptos y no el de los hechos que están conceptualizados. Una razón para estudiar los conceptos de emoción, enojo y otros similares es la amplia utilización que de ellos se hace en psicología. Otra razón es que estos conceptos son procesos psicológicos (y, por tanto, valiosos objetos de estudio) y que interactuan con otros procesos psicológicos. Consideremos, por ejemplo, los conceptos de bruja y de fantasma. Algunas personas, hoy día - y muchas en el pasado- percibían su mundo social en términos de brujas, fantasmas y otras categorías asociadas. Para comprender sus percepciones, emociones, cogniciones y acciones - sus vidas y sus muerteses preciso que entendamos estos conceptos. En forma similar, para comprender las percepciones, cogniciones, emociones y acciones de los miembros de la cultura anglófona actual se requiere que entendamos los conceptos de emoción, enojo y otros parecidos. Y de ello trata el análisis de prototipos.

Esta comprensión no necesariamente nos dice, sin embargo, sobre cómo analizar científicamente los hechos o acontecimientos a los que estas palabras se refieren. Una tarea importante y diferente es prescribir conceptos para el análisis científico de los acontecimientos referidos como emociones. Los conceptos organizados prototípicamente podrían ser útiles en esta tarea. Sin embargo, estoy de acuerdo con Oatley, Johnson-Laird, Ortony, Clore, Foss y otros críticos a la aproximación prototípica, si lo que ellos están planteando es sugerir que nosotros podemos crear conceptos definidos clásicamente que podrían ser útiles en esta tarea. 


\section{Notas}

(1) Parece que la hipótesis del script ha sido mal entendida por Johnson Laird y Oatley, como si se tratara de la pretensión de que una palabra tal como enojo se refiere a los atributos/subacontecimientos especificados por el script. Tal como yo utilizo la noción de script, enojo se refiere a la secuencia completa, y no a los subacontecimientos. Así, enojo no se refiere a la injusticia, que es la causa, ni a la violencia, que es su consecuencia. La hipótesis del script pretende que las causas y consecuencias, junto con otros subacontecimientos, son sin embargo parte del sentido de la palabra enojo. Consideremos la palabra abuela. Cuando uno se entera de que Tina Turner es abuela, uno se entera automáticamente de la existencia de otras personas (dos generaciones de hijos) y de ciertos acontecimientos (nacimientos) que son parte de la palabra abuela; sin embargo, abuela se refiere aquí a Tina Turner y no a su hijo, ni a su nieto, ni al nacimiento de ninguno de ellos. Utilizando el ejemplo de Ryle (1949), la palabra universidad se refiere a un conjunto de facultades, institutos, bibliotecas y residencias etc., pero no a ninguna facultad, instituto, etc., en concreto. La universidad no es algo añadido a las facultades, etc. El enojo no es algo añadido a la injusticia, la violencia, los trastornos fisiológicos, los pensamientos, sentimientos y demás partes del script.

(2) Lyons no intentó elaborar una definición clásica del concepto de emoción sino del de estado emocional. Su esfuerzo deja, pues, en suspenso la cuestión de si la emoción puede definirse clásicamente. Implícitamente, Lyons no está de acuerdo con la afirmación de Ortony et al. según la cual emoción incluye estado como rasgo definitorio:

(3) Clore y Ortony (1988), por ejemplo, afirman: «La meta, sin embargo, no debe ser definir términos emocionales, sino descubrir la estructura de las condiciones psicológicas a las que tales términos se aplican» (p. 391). Por otra parte, Clore y Ortony (1988) también afirman: «El nuestro no es en modo alguno un nuevo concepto de emoción, sino una explicación de lo que se supone que es inherente a los significados existentes de los términos de emoción» (p. 391).

\section{Referencias}

Abelson, R. P. (1981). Psychological status of the script concept. American Psychologist, 36, 715.729.

Armstrong, S. L;., Gleitman, H., Y Gleitman, L. R. (1983). What some concepts might not be. Cognition, 13, 263-308.

Averill, J. R. (1980). A constructivist view of emotion. En R. Plutchik Y H. Kellerman (Eds.), Theories of emotion, Vol. 1. Nueva York: Academic Press.

Averill,J. R. (1982). Anger and aggression: An essay on emotion. Heidelberg: Springer-Verlarg.

BARSALOU, L. W. (1985). Ideals, central tendency, and frequency of instantiation as determinants of graded structure in categories. Journal of Experimental Psychology: Learning, Memory, and Cognition, 11, 629-654.

BARSALOU, L. W. (1987). The instability of graded structure: Implications for the nature of concepts. En U. Neisser (Ed.), Concepts and conceptual development: Ecological and intellectual factors in categorization (pp. 101-140). Cambridge: Cambridge University Press.

Bormann.Kischkel, C; Hildebrand.Pascher, S., Y Stegbauer, G. (1990). The development of emotion concepts: $A$ replication with a German sample. Unpublished manuscript, Max Planck Institute for Psychiatry, Munch, FRG.

Broughton, R. (1984). A prototype strategy for construction of personality scales. Joumal of Personality and Social Psychology, 47, 1.334-1.346.

Bullock, M., Y Russell, J. A. (1986). Concepts of emotion in developmental psychology. En C. E. Izard Y P. B. Read (Eds.), Measuring emotions in infants and children, Vol. II (pp. 203-237). Cambridge: Cambridge University Press.

BuRCh, J. W., Y PIShKIN, V. (1984). Family resemblance - Category structure of joy and shame. Joumal of Clinical Psychology, 40, 1.136-1.143.

Buss, D. M., Y CRAIK, K. H. (1983). The act frequency approach to personality. Psychological Review, 90, 105.126.

CANTOR, N., Y Mischel, W. (1979). Prototypes in person perception. Advances in Experimental Social Psychology, 12, 38-48.

Cantor, N; Mischel, W., Y SChwartz,J. C. (1982). A prototype analysis of psychological situations. Cognitive Psychology, 14, 45-77.

Cantor, N; Smith, E. E; French, R. D., Y Mezzich, J. (1980). Psychiatric diagnosis as prototype categorization. Joumal of Abnormal Psychology, 89, 181-193. 
Chaplin, W. F; John, O. P., Y Goldberg, L. R. (1988). Conceptions of states and traits; Diemsional attributes with ideals as prototypes. Journal of Personality and Social Psychology, $54,541.557$.

Clore, G. L. Y Ortony, A. (1988). The semantics of the affective lexicon. En V. Hamiltion, G. H. Bower Y N. H. Frijda (Eds.), Cognitive perspectives on emotion and motivation (pp. 367-397). Dordrecht: Kluwer.

Conway, M. A. (in press). Conceptual representation of emotions: The role of autobiogra phical memories. En Gilhooly, Keane, Logie, Y Erbos (Eds.), Lines of thinking: Reflections on the psychology of thought. Wiley.

Conway, M. A., Y Bexerian, D. A. (1987). Situational knowledge and emotions. Cognition and Emotion, 1, 145-191.

Dufry, E. (1934). Emotion: An example of the need for reorientation in psychology. Psychological Review, 41, 184-198.

DufFy, E. (1941). An explanation of «emotional» phenomena without the use of the concept «emotion» The Journal of General Psychology, 25, 283-293.

Exman, P; Friesen, W. V., YSimons, R. C. (1985). Is the startle reaction an emotion? Journal of Personality and Social Psychology, 49, 1.416-1.426.

Ferh, B. (1982). Prototype categorization of emotion. Unpublished master's thesis, University of British Columbi, Vancouver, Canadá.

FerHR, B. (1988). Prototype analysis of the concepts of love and commitment. Joumal of Personality and Social Psychology, 55, 557-579.

FeHR, B., Y Russell, J. A. (1984). Concepts of emotion viewed from a prototype perspective. Joumal of Experimental Psychology: General, 113, 464-486.

FEHR, B., Y RusselL, J. A. (1989). The concept of love viewed from a prototype perspective. Unpublished manuscript. University of Winnipeg.

Fetr, B., Y Russell, J. A., Y W Ard, L. M. (1982). Prototypicality of emotions: A reaction time study. Bulletin of the Psychonomic Society, 20, 253-254.

Gurtman, M. B. (1987, August). What is an affective prototype? Paper presented at the annual meeting of the American Psychological Association, Nueva York.

HARNAD, S. (1987). Category induction and representation. En S. Harnad (Ed.), Categorical perception (pp. 535-565). Cambridge: Cambridge University Press.

Horowitz, L. M; Wright, J. C; Lowenstein, E., Y Parad, H. W. (1981). The prototype as a construct in abnormal psychology: I. A method for deriving prototypes. Journal of $A b$ normal Psychology, 90, 586-574.

Hume, D. (1978). A treatise of buman nature. In L. A. Selby-Bigge (Ed.). Oxford: Oxford University Press. (Original work published, 1.739-1.740)

IACCINO, V. (1989). Understanding the concept emotion: Support for a prototype perspective. ERIC-CAPS: Office of Educational Research and Improvement, Communication and Reading Skills Database, \# ED299-637, filmed April 13, 1989, 90 pp. (On microfiche).

Izard, C. E. (1977). Human emotions. Nueva York: Plenum Press.

James, W. (1950) The principles of psychology. Nueva York Dover. (Original work published, $1.890)$

JAMES, W. (1929). The varieties of religious experience: $A$ study in buman nature. Nueva York: Longmans, Green y Co. (Original work published, 1.902).

Johnson.Laird, P.N., Y Oatley, K. (1986). The meaning of emotions: A cognitive theory and a semantic analysis. Unpublished manuscript. Cambridge University.

Johnson-LAird, P. N., Y OATLEY, K. (1988). Il significato delle emozioni: una teoria e un'analisi semantica. En V. D’Urso e R. Trentin (Eds.), Psicologia delle emozioni (pp. 119-158). Bologna: Il Mulino.

Johnson-Laird, P. N., Y OATLey, K. (1989). The language of emotions: An analysis of a semantic field. Cognition and Emotion, 3, 81-123.

KAGAN, J. (1978). On emotion and its development: A working paper. En M. Lewis y L. A. Rosenblum (Eds.), The development of affect (pp. 11-42). Nueva York: Plenum.

Kahneman, D. Y Miller, D. T (1986). Norm theory: Comparing reality to its alternatives. Psychological Review, 93, 136-153.

KAY, P., Y McDANIEL, C. (1978). The linguistic significance of the meanings of basic color terms. Language, 54, 610-646.

LABOv. W. (1973). The boundaries of words and their meanings. En C. J. N. Bailey y R. W. Shuy (Eds.), New ways of analysing variation in English (pp. 340-373). Washington, DC: Georgetown University Press.

LAKOFF, G. (1987). Women, fire, and dangerous things: What categories reveal about the mind. Chicago: University of Chicago Press.

LuTz, C. (1985). Cultural patterns and individual differences in the child's emotional meaning system. En M. Lewis y C. Saarni (Eds.), The socialization of emotions (pp. 37.53). Nueva York: Plenum. 
Lyons, W. (1980). Emotion. Cambridge: Cambridge University Press.

Lysak, H; Rule, B. G., Y DobBs, A. R. (1989). Conceptions of aggression: Prototype or definign features? Personality and Social Psychology Bulletin, 15, 233.243.

Mascolo, M. F., Y Mancuso, J. C. (1988). The structure and content of emotion knowledge: A prototype approach. Manuscript submitted for publication.

Medin, D. L. (1989). Concepts and conceptual structure. American Psychologist, 44, 1.469-1.481.

Mervis, C. B., Y Rosch, E. (1981). Categorization of natural objetcs,. Annual Review of psychology, 32, 89-115.

NEISSER, U. (1979). The concept of intelligence. Intelligence, 3, 217-227.

NeISSER, U. (1987). Concepts and conceptual development: Ecological and intellectual factors in categorization. Cambridge: Cambridge University Press.

Ortony, A; Clore, G. L., Y Foss, M. A. (1987). The referential structure of the affective lexicon. Cognitive Science, 11, 341-364.

Osherson, D. N., Y S Sith, E. E. (1981). On the adequacy of prototype theory as a theory of concepts. Cognition, 9, 35-58.

Panksepp, V. (1982). Toward a general psychobiological theoy of emotions. Bebavioral and Brain Sciences, 5, 407.467.

Posner, M. I., Y Keele, S. W. (1968). On the genesis of abstract ideas. Joumal of Experimental Psychology, 77, 353-363.

Rombouts, H. (1988). Emoties gezien vanuit een prototype-perspectief. En G. Mulder \& R. J. Takens (Eds.), Nederlands Psychologisch Onderzoek I: Bijdragen aan de klinische psychologie en bet emotie-onderzoek (pp. 37-50). Amsterdam/Lisse: Swets \& Zeitlinger.

Rosch, R. (1977). Human categorization. En N. Warren (Ed.), Studies in cross-cultural psychology (pp. 1-49). Londres: Academic Press.

RusselL, J. A. (1989). Culture, scripts, and children's understanding of emotion. En C. Saarni y P. Harris (Eds.), Children's understanding of emotion (pp. 293-318). Cambridge: Cambridge University Press.

Russell, J. A., Y Bullock, M. (1986). Fuzzy concepts and the perception of emotion in facial expressions. Social Cognition, 4, 309-341.

RusSELL, J. A., Y FEHR, B. (1989). The concept of anger viewed from a prototype perspective. Unpublished manuscript, University of British Columbia.

RYLE, G. (1949). The concept of mind. Nueva York: Penguin Books.

Schachter, S., Y Singer, J. E. (1962). Cognitive, social, and physicological determinants of emotional state. Psychological Review, 69, 379-399.

Shaver, P; Schwartz, J; Kirson, D; O'Connor, C. (1987). Emotion knowledge: Further exploration of a prototype approach. Journal of Personality and Social Psychology, 52, 1.061-1.086.

SHIELDS, S. A. (1984). Distinguishing between emotion and nonemotion: Judgments about experience. Motivation and Emotion, 8, 335-369.

Smith, E. E., Y Medin, D. L. (1981). Categories and concepts. Cambridge, MA: Harvard University Press.

Tiller, D. K., Y Harris, P. L. (1984). Prototypicality of emotion concepts: A discussion of normative data. Unpublished manuscript, Oxford University.

Tversky, B., Y Hemenway, K. (1983). Categories of environmental scenes. Cognitive Psycbo$\log y, 15,121-149$.

W IerZBICKA, A. (1972). Semantic primitives. Frankcfort: Athenum.

WIERZBICKA, A. (1973). The semantic structure of words for emotions. En R. Jakobson, D. H. van Schooneveld, y D. S. Worth (Eds.), Slavic poetics: Essays in honor of Kiril Taranovsky. The Hague: Mouton.

WiERZBiCKA, A. (1984). Cups and mugs: Lexicography and conceptual analysis. Australian Joumal of Linguistics.

WitTgenstein, L. (1953). Philosophical investigations. Nueva York: Macmillan.

ZADEH, L. A. (1965). Fuzzy sets. Information and control, 8, 338-353.

Zajonc, R. B. (1980). Feeling and thinking: Preferences need no inferences. American Psychologist, 35, 151-175. 